\title{
Chitosan temperature-sensitive gel loaded with drug microspheres has excellent effectiveness, biocompatibility and safety as an ophthalmic drug delivery system
}

\author{
XIAOYING KONG $^{1 *}$, WENHUA XU ${ }^{2 *}$, CUIPING ZHANG ${ }^{3}$ and WEI KONG ${ }^{4}$ \\ ${ }^{1}$ Department of Material Chemistry, College of Chemistry and Pharmaceutical Science, Qingdao Agricultural University, \\ Qingdao, Shandong 266109; ${ }^{2}$ Department of Inspection, Medical College, Qingdao University, Qingdao, \\ Shandong 266000; ${ }^{3}$ Emergency Department, Shandong Energy Zaozhuang Mining Group Hospital, Zaozhuang, \\ Shandong 277000; ${ }^{4}$ Department of Cardiology, Lianhe Hospital, Dezhou, Shandong 253000, P.R. China
}

Received December 9, 2016; Accepted September 13, 2017

DOI: $10.3892 /$ etm.2017.5586

\begin{abstract}
In the present study, a temperature-sensitive gel composed of chitosan, carboxymethyl chitosan and glycerophosphate was prepared and loaded with chitosan microspheres encapsulating levofloxacin. The bioavailability of levofloxacin and the safety of this novel opthalmic drug delivery formulation were evaluated. Levofloxacin chitosan microspheres were prepared using the ionic gelation method, and the particle size and entrapment rate were determined. The morphology of the microspheres was observed by scanning electron microscopy. The $\mathrm{pH}$ and zeta potential were measured. The in vitro release of levofloxacin by the chitosan temperature-sensitive gel loaded with drug microspheres was determined using spectrophotometry. The eye retention time of the chitosan temperature-sensitive gel was calculated using a fluorescein sodium test. To assess the bioavailability and safety of the chitosan temperature-sensitive gel, a cell compatibility test, a cytotoxicity test and skin irritation test were performed. The entrapment rate of levofloxacin in the chitosan microspheres was determined to be $26.5 \%$. The levofloxacin chitosan microspheres that were formed by chitosan and sodium tripolyphosphate were identified to be suitable for use in an ophthalmic particle dispersion system based on their physical and chemical properties. The $\mathrm{pH}$ of the levofloxacin chitosan microsphere suspension was $5.87 \pm 0.04$, the average particle diameter was $2,452 \pm 342 \mathrm{~nm}$,
\end{abstract}

Correspondence to: Dr Xiaoying Kong, Department of Material Chemistry, College of Chemistry and Pharmaceutical Science, Qingdao Agricultural University, 700 Changcheng Road, Qingdao, Shandong 266109, P.R. China

E-mail: xykong51@aliyun.com

${ }^{*}$ Contributed equally

Key words: chitosan, temperature-sensitive gel, drug microsphere, ophthalmic drug delivery system, biocompatibility the polydispersity index was $0.168 \pm 0.028$ and the $\zeta$ potential was $28.62 \pm 1.7 \mathrm{mV}$. The chitosan temperature-sensitive gel carrying microspheres loaded with drug prevented drug burst release at the initial stage and facilitated the slow release of the drug later on. Furthermore, this delivery system markedly prolonged the contact duration of levofloxacin with the eye. The chitosan temperature-sensitive hydrogel was safe and provided a good bioavailability of the drug. The results revealed that the chitosan temperature-sensitive gel had a cytotoxicity of grade 0 , and no erythematous response was observed during the entire course of the skin irritation test. The present study provided a basis for the future development of the chitosan-based temperature-sensitive hydrogel in ophthalmic drug delivery.

\section{Introduction}

Eye drops account for $\sim 90 \%$ of ophthalmic drug delivery systems available (1). Traditional eye drops are usually lost from the ocular surface by tear washing or nasolacrimal drainage immediately after administration, with poor ocular bioavailability and numerous adverse reactions (2). In addition, due to the inconvenience of night administration, the pharmacological peak-valley phenomenon is prominent, greatly affecting the treatment effect of ocular drugs. With the development of novel technologies, novel ophthalmic drug delivery systems have been designed to effectively elevate ocular bioavailability by prolonging the retention time of drugs in the eye or improving the penetration of drugs into the cornea and conjunctiva $(3,4)$.

Chitosan is a natural cationic polysaccharide that is prepared from chitin after partial or complete deacetylation. Due to its excellent biological properties, chitosan and its derivatives have been widely used in ophthalmic drug delivery systems (5). As a novel form of administration, in situ gel undergoes phase transition to a non-chemically crosslinked semi-solid gel preparation at the medication site immediately after administration in solution or semi-solid format. In situ gel prepared from chitosan or its derivatives forms a transparent gel that has a strong affinity to the ocular surface under ocular 
physiological $\mathrm{pH}$ conditions. Certain chitosan derivatives are temperature-sensitive and may be prepared to form a temperature-sensitive gel in situ (6,7). The temperature-sensitive gel formation is due to changes in the physical state of polymers induced by changes in hydrogen bonds or hydrophobic interactions (8-11). Cao et al (12) synthesized a novel type of isopropyl acrylamide chitosan polymer and used it to prepare a timolol maleate temperature-sensitive in situ gel, which provides a higher bioavailability than its respective solution.

At present, certain challenges remain regarding the development of in situ gels for pharmaceutical application. First, higher concentrations of polymers and non-aqueous solvent in the gel may cause eye irritation and safety issues. Furthermore, there may be a drug burst between the time-points of dripping the formulation into the eye and the formation of the gel, leading to a significant peak-valley phenomenon $(13,14)$. In the present study, a temperature-sensitive gel was prepared using chitosan, carboxymethyl chitosan and glycerophosphate, and levofloxacin was encapsulated into chitosan microspheres, which were embedded in the chitosan temperature-sensitive gel. In addition, the safety of chitosan temperature-sensitive gel carrying drug microspheres and the bioavailability they provided were evaluated.

\section{Materials and methods}

Preparation of chitosan microspheres. Chitosan acetic acid solution (2.0 g/1; Biotemed, Qingdao, China) was prepared and adjusted to $\mathrm{pH}$ 5.0. Subsequently, $20 \mathrm{mg}$ levofloxacin (Wuhan DKY Technology, Wuhan, China) was dissolved in $100 \mathrm{ml}$ chitosan acetic acid solution with agitation at 10.06-13.42 x g. Subsequently, sodium tripolyphosphate $(1.0 \mathrm{~g} / \mathrm{l}$; Tianjin Bodi Chemical, Co., Ltd., Tianjin, China) was added to obtain a suspension after agitation for $15 \mathrm{~min}$. Levofloxacin chitosan microspheres $(2.4 \mathrm{~g} / \mathrm{l})$ were then obtained by filtration.

Determination of entrapment rate of levofloxacin chitosan microspheres. A $200 \mathrm{mg} / \mathrm{l}$-stock solution of levofloxacin (20 $\mathrm{mg}$ ) in $0.1 \mathrm{M}$ acetic acid solution was prepared. A series of solutions with mass concentrations of levofloxacin of $4,6,8,10,12,14,16$ and $18 \mathrm{mg} / \mathrm{l}$ were prepared and the absorbance at each concentration was measured at $293 \mathrm{~nm}$ (UV2 9200; Nanjing Xinhang Scientific Instrument Co., Ltd., Nanjing, China). Levofloxacin chitosan microspheres $(2.0 \mathrm{ml})$ were centrifuged at $671 \mathrm{x} \mathrm{g}$ at $4^{\circ} \mathrm{C}$ for $30 \mathrm{~min}$. Supernatants were obtained and diluted with $0.1 \mathrm{M} \mathrm{HCl}$. The concentration of free levofloxacin $\left(\mathrm{C}_{\text {free }}\right)$ in the suspension was determined. In the meantime, levofloxacin chitosan microspheres $(2.0 \mathrm{ml}$; $2.4 \mathrm{~g} / \mathrm{l})$ which was the reaction solution from above were suspended in $8.0 \mathrm{ml} 0.1 \mathrm{~mol} / 1 \mathrm{HCl}$ solution and incubated at $60^{\circ} \mathrm{C}$ for $1 \mathrm{~h}$ for hydrolysis. After filtration using a microfiltration membrane (pore size, $0.02 \mu \mathrm{m}$; EMD Millipore, Billerica, MA, USA), the total concentration of levofloxacin in the filtrate $\left(\mathrm{C}_{\text {total }}\right)$ was determined. The entrapment rate of levofloxacin chitosan microspheres was calculated using the following equation: Entrapment rate $=\left(1-\mathrm{C}_{\text {free }} / \mathrm{C}_{\text {total }}\right) \times 100 \%$. Each test was repeated for 5 times.

Characterization of the physical and chemical properties of levofloxacin chitosan microspheres. A suspension of levofloxacin chitosan microspheres was used to determine the $\mathrm{pH}, \zeta$ potential, particle diameter (Zetasizer $3000 \mathrm{HSa}$; Malvern Instruments, Malvern, UK) and particle size distribution. After diluting the suspension of levofloxacin chitosan microspheres, a scanning electron microscope (JEOL7500F; Jeol, Tokyo, Japan) was used to observe the morphology of the particles. In addition, an infrared spectrum was used to analyze pure chitosan powder, blank chitosan microspheres and levofloxacin chitosan microspheres. The scanning range was $400-4,000 \mathrm{~cm}^{-1}$ and the resolution was $4 \mathrm{~cm}^{-1}$.

Preparation of chitosan-carboxymethyl chitosan temperature-sensitive gel loaded with chitosan nanoparticles. Sodium glycerophosphate $(0.78 \mathrm{~g})$ and carboxymethyl chitosan $(0.2 \mathrm{~g})$ were dissolved in 2 and $3 \mathrm{ml}$ double-distilled $(\mathrm{dd}) \mathrm{H}_{2} \mathrm{O}$, respectively. Furthermore, chitosan $(0.2 \mathrm{~g})$ was dissolved in $5 \mathrm{ml} 0.1 \mathrm{M}$ acetic acid. With mixing, sodium the glycerophosphate solution and carboxymethyl chitosan solution were consecutively added to the chitosan solution to thereby obtain the chitosan-carboxymethyl chitosan temperature-sensitive gel system. Subsequently, $0.1 \mathrm{~g}$ levofloxacin chitosan microspheres were added to $10 \mathrm{ml}$ chitosan-carboxymethyl chitosan temperature-sensitive gel, followed by thorough mixing.

Evaluation of in vitro release of levofloxacin by chitosan temperature-sensitive gel loaded with drug microspheres. In the different experimental groups, the drug delivery systems were prepared via three different methods as follows: Group 1, levofloxacin was added into chitosan-carboxymethyl chitosan solution; group 2, levofloxacin was added into chitosan-carboxymethyl chitosan-sodium glycerophosphate solution; group 3, temperature-sensitive gel containing levofloxacin nanoparticles was prepared as described above. The liquid samples were put into dialysis bags (molecular weight cut-off, 7,000 Da), which were soaked in $200 \mathrm{ml}$ phosphate-buffered saline ( $\mathrm{pH} 7.4)$ at $37^{\circ} \mathrm{C}$. After standing still for $20 \mathrm{sec}, 5 \mathrm{ml}$ phosphate buffer solution was transferred to a sample tube. After the formation of a solid gel in dialysis bag at $37^{\circ} \mathrm{C}, 5 \mathrm{ml}$ phosphate buffer solution was also transferred to individual sample tubes at 5, 10, 15, 20, 30, 60, 120, 180, 240, $300,360,420,480,540,600,660$ and $720 \mathrm{~min}$. Each time after withdrawal of phosphate buffer solution, another $5 \mathrm{ml}$ of fresh solution was replenished. For group 1, in which no gel was formed, phosphate buffer solution was also aspirated at the abovementioned time-points. The absorbance of levofloxacin in each sample tube was measured at 293 nm (UV2 9200; Nanjing Xinhang Scientific Instrument Co., Ltd.). Each sample was tested in triplicate. Using the levofloxacin standard curve, the concentrations of levofloxacin from each group at the different time-points were calculated and levofloxacin in vitro release curves were plotted.

Eye retention test of chitosan temperature-sensitive gel. The experimental animal protocols of the present study were approved by the Ethics Committee of Qingdao Agricultural University (Qingdao, China). On ice, fluorescein sodium (mass fraction, $0.05 \%$ ) was added into a levofloxacin drug loading system in groups 1-3. A total of 18 healthy New Zealand white rabbits (Qingdao Medicine Inspecting Institute, Qingdao, 
China; age, 8 months; weight, $1.5-2.0 \mathrm{~kg}$ ) were divided into 3 groups of 6 rabbits. Rabbits had free access to food and water in a temperature-controlled environment of $25^{\circ} \mathrm{C}(45-65 \%$ humidity) and a 12-h light/dark cycle. Temperature-sensitive gel in pure form was dripped into the conjunctival sac on the left side, and the eye on the right side was used as a blank control. After drug administration, the eyes were closed under local anesthesia in accordance with experimental animal welfare guidelines. The continuous fluorescent layer of the corneal surface was observed under a slit lamp at regular intervals. The time of disappearance of the continuous fluorescent layer on the corneal surface following application of the gel was considered to be the ocular retention time of levofloxacin

Cell compatibility test. The chitosan-based temperature-sensitive hydrogel was prepared as a preparation of chitosan-carboxymethyl chitosan temperature-sensitive gel loaded with chitosan nanoparticles, and the thickness of the hydrogel was controlled at 1-2 mm, which could be regarded as a membrane. The membrane was cut into circular sheets with a diameter of $1.1 \mathrm{~cm}$. The circular sheets were soaked in 25, 50 and $75 \%$ ethanol for $2-3 \mathrm{~h}$ each time and then with $75 \%$ ethanol for 1-2 days, followed by transfer to sterile D-Hank's buffer for soaking over $10 \mathrm{~h}$ with the buffer being replaced 4-5 times. The membranes were then soaked in D-Hank's buffer prior to use. The membranes were placed into 48 -well cell culture plates containing $100 \mu \mathrm{l}$ Dulbecco's modified Eagle's medium (DMEM; Tianjin Bodi Chemicals, Tianjin, China) supplemented with $10 \%$ fetal bovine serum. Each time-point had 5 repeats. Subsequently, rabbit corneal endothelial cells (15) were trypsinized and adjusted to a concentration of $5 \times 10^{5} / \mathrm{ml}$. Cell suspension $(200 \mu \mathrm{l})$ was added into predefined wells. The negative control contained DMEM only. The cells were cultured at $37^{\circ} \mathrm{C}$ and $5 \% \mathrm{CO}_{2}$. On days 3 and 5 , the cells were observed and images were captured. The cells were then trypsinized and transferred to 96 -well plates, followed by incubation over $12 \mathrm{~h}$. Subsequently $5 \mathrm{mg} / \mathrm{ml}$ MTT $(20 \mu \mathrm{l})$ was added to each well, followed by further incubation for $4 \mathrm{~h}$. After discarding the medium, dimethyl sulfoxide (DMSO; $150 \mu \mathrm{l}$ ) was added and the wells were incubated at $37^{\circ} \mathrm{C}$ for $10 \mathrm{~min}$. After agitation for $40 \mathrm{sec}$, the absorbance of each well was measured at $490 \mathrm{~nm}$ on an UV2 9200 plate reader (Nanjing Xinhang Scientific Instrument Co., Ltd.).

Cytotoxicity test. The L929 mouse fibroblast cell line at the logarithmic growth phase was trypsinized, the suspension was adjusted to a concentration of $1 \times 10^{4} / \mathrm{ml}$, and the cells were seeded onto 96-well plates (200 $\mu \mathrm{l} /$ well). After incubation at $37^{\circ} \mathrm{C}$ and $5 \% \mathrm{CO}_{2}$, the medium was replaced with DMEM that had been used to soak the chitosan-based temperature-sensitive hydrogel membrane for $24 \mathrm{~h}$ in the experimental group. For the control group, normal medium was added. Each group had 12 repeats. On days 3 and 5, the cells were observed under a microscope. Subsequently, $20 \mu \mathrm{l}$ MTT solution $(5 \mathrm{mg} / \mathrm{ml})$ was added into each well and the cells were incubated for $4 \mathrm{~h}$ under normal culturing conditions. After discarding the medium, DMSO $(150 \mu \mathrm{l})$ was added, followed by incubation at $37^{\circ} \mathrm{C}$ for $10 \mathrm{~min}$. After shaking for $40 \mathrm{sec}$, the absorbance of each well was measured at $490 \mathrm{~nm}$ (UV2 9200; Nanjing Xinhang Scientific Instrument Co., Ltd.). The relative
Table I. ISO 10993.10-2002 scoring standard.

\begin{tabular}{lccc}
\hline ER & Grade & Edema response & Grade \\
\hline None & 0 & None & 0 \\
Mild & 1 & Mild & 1 \\
Clear & 2 & Clear & 2 \\
Moderate & 3 & Moderate & 3 \\
Severe ER or escharosis & 4 & Severe & 4 \\
\hline
\end{tabular}

ED, edema; ER, erythematous response.

growth rate $(\mathrm{RGH})$ was calculated as follows: $\mathrm{RGH}=$ mean absorbance in experimental group/mean absorbance in control group x $100 \%$. Cytotoxicity grading was as follows: $0, \mathrm{RGH} \geq 100 \% ; 1,75 \%<\mathrm{RGH}<95 \% ; 2,50 \%<\mathrm{RGH}<74 \%$; $3,25 \%<\mathrm{RGH}<49 \%$; 4, $1 \%<\mathrm{RGH}<24 \%$; and $5, \mathrm{RGH}=0 \%$. For grade 1 , the cytotoxicity was considered acceptable; for grade 2 , cytotoxicity was evaluated in combination with cell morphological analysis; for grades 3-5, cytotoxicity was considered unacceptable.

Skin irritation test. Sterile and pyrogen-free chitosan-based temperature-sensitive hydrogel membrane (5 g) was soaked in $5 \mathrm{ml}$ saline for $72 \mathrm{~h}$. At $4-24 \mathrm{~h}$ prior to the experiments, the hair of three healthy rabbits was removed on both sides of the dorsal spine. Six points were selected on each side of the dorsal spine. The six points on the left side were included in the control group and those on the right side were included in the experimental group. Saline $(0.1 \mathrm{ml})$ was injected subcutaneously into the six points of the control group, while chitosan-based temperature-sensitive hydrogel membrane extract liquid $(0.1 \mathrm{ml})$ that was DMEM soaked with the gel for $24 \mathrm{~h}$, was injected subcutaneously into the six points of the experimental group. At 12, 24 and $48 \mathrm{~h}$ after injection, each injection point of each rabbit was evaluated according to Table I to determine the artificial vascular stimulation fraction. On days 3, 5 and 7 after surgery, subcutaneous tissues were obtained from all animals under anesthesia with $1 \mathrm{mg} / \mathrm{kg}$ sodium pentobarbital and subjected to hematoxylin and eosin (H\&E) staining. The samples were paraffin-embedded, sliced, and washed with xylene for 15 min twice, $100 \%$ ethanol for $5 \mathrm{~min}$ twice, $80 \%$ ethanol for $5 \mathrm{~min}$ once, and $\mathrm{ddH}_{2} \mathrm{O}$ for $5 \mathrm{~min}$ once, followed by staining with hematoxylin at $25^{\circ} \mathrm{C}$ for $5 \mathrm{~min}$. After washing with water for 1-3 sec, the samples were washed with $1 \%$ hydrochloric acid $(1 \mathrm{ml})$ and $75 \%$ ethanol solution (99 ml) for 1-3 sec prior to washing with water for 10-30 sec. After washing with $\mathrm{dd}_{2} \mathrm{O}$ for $1-2 \mathrm{sec}$, the samples were stained using $0.5 \%$ eosin at $25^{\circ} \mathrm{C}$ for $1-3 \mathrm{~min}$. Subsequently, the samples were rehydrated/dehydrated in a graded ethanol series. The samples were then washed with xylene for three times of 2 min and mounted with neutral balsam.

Statistical analysis. The results were analyzed using SPSS v17.0 software (SPSS, Inc., Chicago, IL, USA). Values are expressed as the mean \pm standard deviation. Differences were compared using Student's t-test. $\mathrm{P}<0.05$ was considered to indicate a statistically significant difference. 
Table II. Entrapment rate of levofloxacin chitosan microspheres $(n=5)$.

\begin{tabular}{lccc}
\hline $\mathrm{C}_{\text {free }}(\mathrm{g} / \mathrm{l})$ & $\mathrm{C}_{\text {total }}(\mathrm{g} / \mathrm{l})$ & Entrapment rate $(\%)$ & Mean $\pm \mathrm{RSD}$ \\
\hline 0.312 & 0.425 & 26.6 & \\
0.305 & 0.418 & 27.0 & $26.5 \pm 1.31 \%$ \\
0.319 & 0.432 & 26.2 & \\
0.314 & 0.429 & 26.8 & \\
0.322 & 0.436 & 26.1 & \\
\hline
\end{tabular}

$\mathrm{C}_{\text {free }}$, concentration of free chitosan; $\mathrm{C}_{\text {total }}$, maximum concentration of chitosan that can be released from levofloxacin chitosan microspheres; RSD, relative standard deviation.

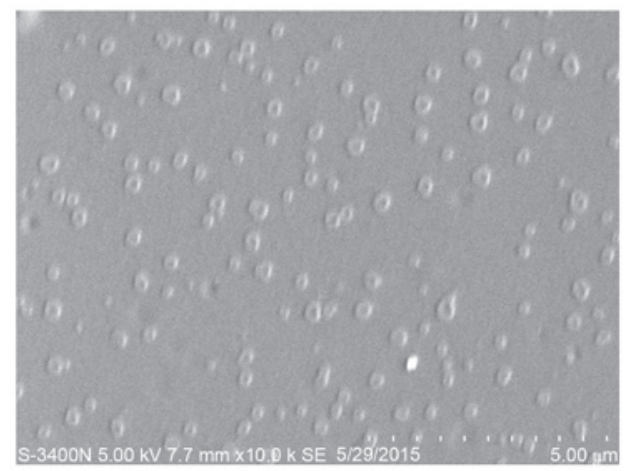

Figure 1. Scanning electron microscopy image of levofloxacin chitosan microspheres. After diluting the suspension of levofloxacin chitosan microspheres, a scanning electron microscope (JEOL7500F; Jeol) was used to observe the morphology of particles (magnification, x10,000).

\section{Results}

Levofloxacin chitosan microspheres have an entrapment rate of $26.5 \pm 1.31 \%$. To determine the entrapment rate of levofloxacin chitosan microspheres, the measurement data were subjected to regression analysis, resulting in the following standard equation: $\mathrm{A}=0.08752+59.951 \mathrm{C}(\mathrm{r}=0.9998$ when $\mathrm{C}<100 \mathrm{mg} / \mathrm{l})$ with $\mathrm{A}$ being the absorbance and $\mathrm{C}$ the mass concentration (mg/l). The measurement data were the absorbance values of the levofloxacin standard solutions at different concentrations. The linear range of the standard curve was 6-16 mg/l. Using this equation, the concentration of each diluted sample was calculated and then converted to the concentrations prior to dilution to obtain $\mathrm{C}_{\text {free }}$ and $\mathrm{C}_{\text {total }}$. The mean entrapment rate of the levofloxacin chitosan microspheres was calculated from the measurement data of five experiments (Table II). The result suggested that the entrapment rate of levofloxacin chitosan microspheres was $26.5 \pm 1.31 \%$.

Levofloxacin chitosan microspheres formed by chitosan and sodium tripolyphosphate have physical and chemical properties rendering them a suitable ophthalmic particle dispersion system. To characterize the levofloxacin chitosan microspheres regarding their physical and chemical properties, the $\mathrm{pH}$ and $\zeta$ potential, as well as the particle diameter and distribution were determined, and a scanning electron microscope was

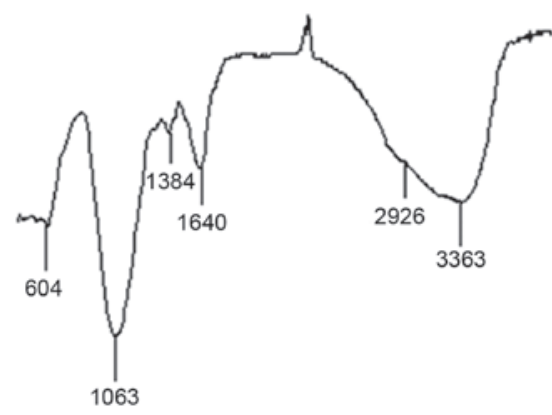

$\begin{array}{lllllllll}500 & 1000 & 1500 & 2000 & 2500 & 3000 & 3500 & 4000 & 4500\end{array}$ $\left(\mathrm{cm}^{-1}\right)$

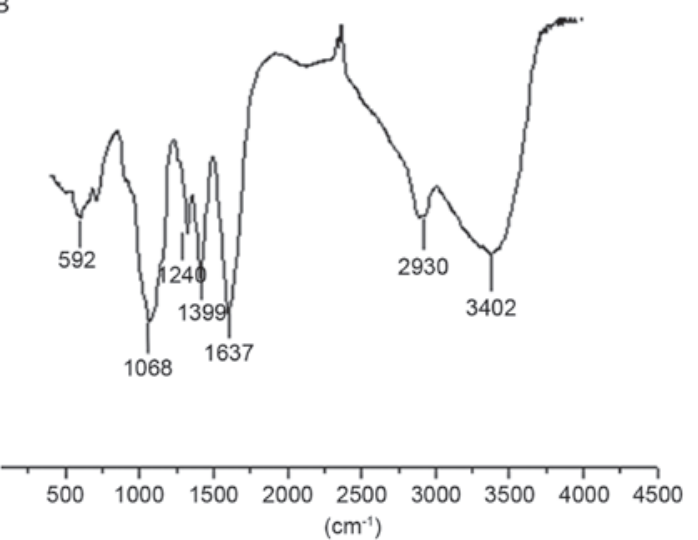

Figure 2. Infrared spectrum of (A) chitosan and (B) levofloxacin chitosan microspheres. The scanning range was $400-4,000 \mathrm{~cm}^{-1}$ and the resolution was $4 \mathrm{~cm}^{-1}$.

used to observe the morphology of the particles. In addition, an infrared spectrum was recorded to analyze pure chitosan powder, blank chitosan microspheres and levofloxacin chitosan microspheres. The results indicated that the $\mathrm{pH}$ of levofloxacin chitosan microsphere suspension was $5.87 \pm 0.04$, the average particle diameter was $2,452 \pm 342 \mathrm{~nm}$, the polydispersity index was $0.168 \pm 0.028$ and the $\zeta$ potential was $28.62 \pm 1.7 \mathrm{mV}$. Scanning electron microscopy revealed that the shape of the levofloxacin chitosan microspheres was almost spherical (Fig. 1). The infrared spectrum demonstrated the existence of chitosan by intense absorbance peaks at $1,063 \mathrm{~cm}^{-1}(\mathrm{C}-\mathrm{O}$ in hydroxyl or cyclic ether) and $3,363 \mathrm{~cm}^{-1}(\mathrm{O}-\mathrm{H}$ and $\mathrm{N}-\mathrm{H}$ of polysaccharides) (Fig. 2A). In addition, the formation of microspheres by chitosan and sodium tripolyphosphate was indicated by peaks at $1,399 \mathrm{~cm}^{-1}$ (amino acid cross-linked by ion) and $592.9 \mathrm{~cm}^{-1}$ (interaction between phosphate and chitosan amino site) (Fig. 2B). These results indicate that levofloxacin chitosan microspheres that are formed by chitosan and sodium tripolyphosphate are suitable for use as an ophthalmic particle dispersion system due to their physical and chemical properties.

Chitosan temperature-sensitive gel containing microspheres loaded with drug prevent drug burst release at the initial stage and facilitates the slow release of the drug later on. To evaluate the in vitro release of levofloxacin by the chitosan temperature-sensitive gel, three different groups were used with different sample preparations. In group 1, a great amount of 


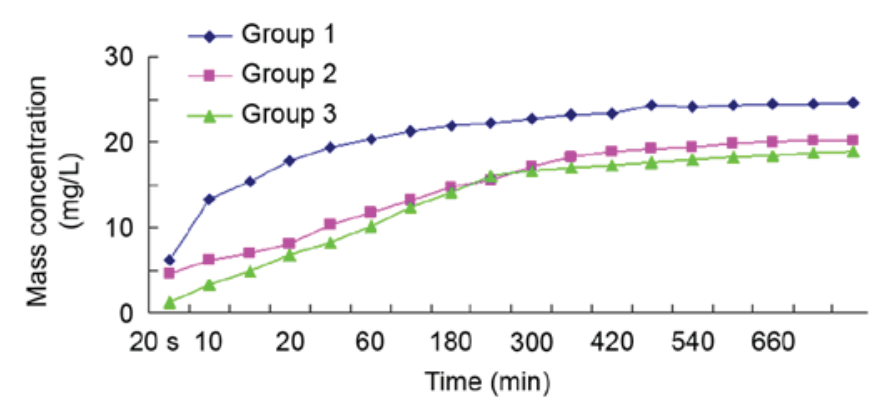

Figure 3. In vitro release of levofloxacin. The formulations in groups 1-3 were prepared as follows: Group 1, levofloxacin was added into a chitosan-carboxymethyl chitosan solution; group 2, levofloxacin was added into a chitosan-carboxymethyl chitosan-sodium glycerophosphate solution; group 3, a temperature-sensitive gel containing levofloxacin nanoparticles was prepared.
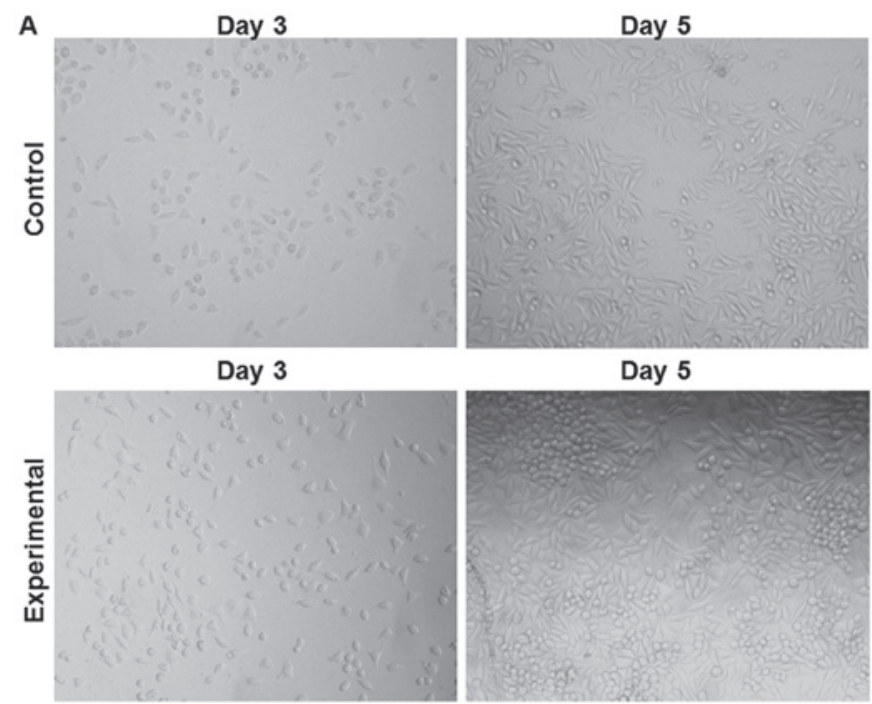

Day 5

B

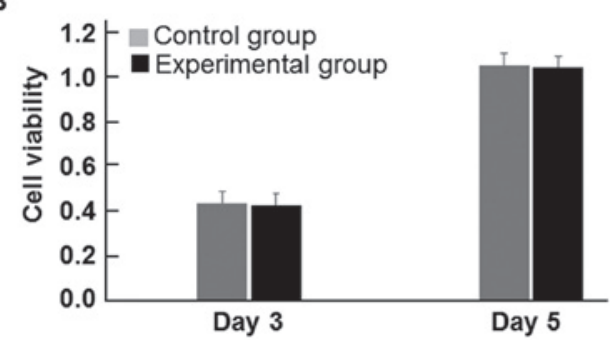

Figure 4. Cell compatibility test. (A) Morphology of corneal endothelial cells on chitosan temperature-sensitive gel membrane in control and experimental groups on days 3 and 5 (magnification, x100). (B) MTT analysis of cytocompatibility of corneal endothelial cells grown on a chitosan temperature-sensitive gel membrane on days 3 and 5 .

drug was released at the initial $20 \mathrm{sec}$ and the drug burst release was dramatic within the first $10 \mathrm{~min}$. For group 2, marked drug release was also observed within the first $20 \mathrm{sec}$, but later on, the drug was slowly released with time. For group 3, the slow release of the drug was similar to that in group 2 after $15 \mathrm{~min}$, but the burst release of the drug was effectively prohibited within the first $15 \mathrm{~min}$ (Fig. 3). These results suggest that chitosan temperature-sensitive gel containing microspheres loaded with drug prevents drug burst release at the initial stage and facilitates the slow release of the drug later on.
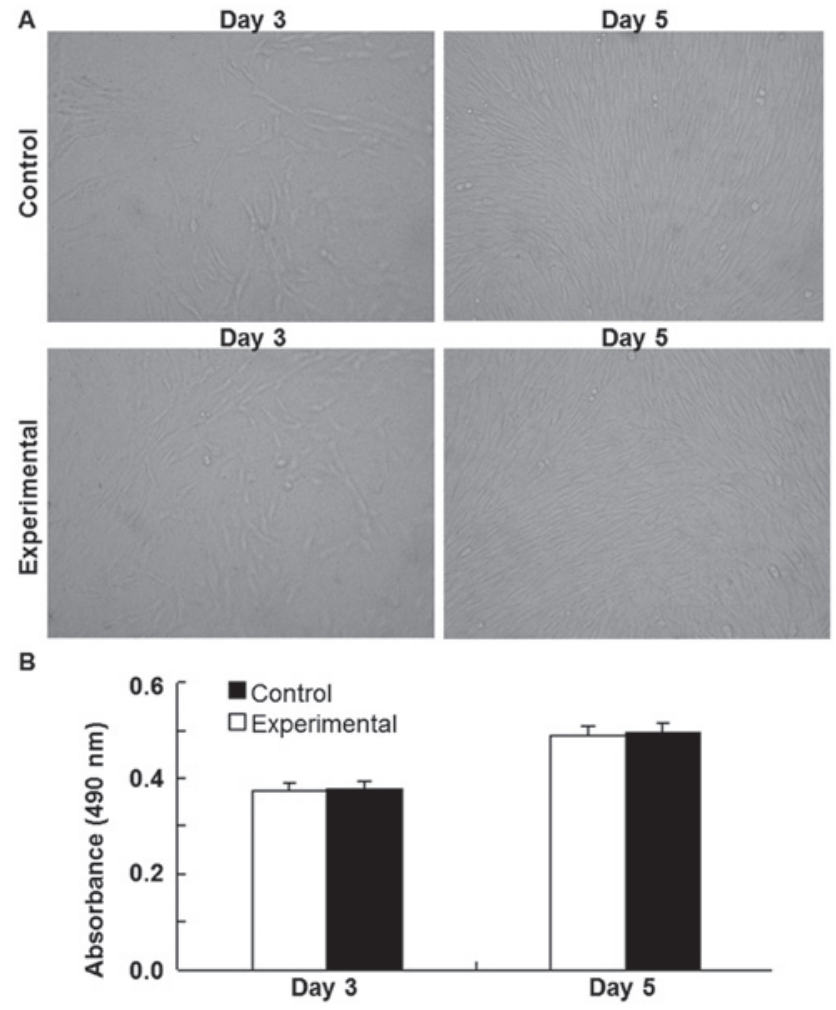

Figure 5. Cytotoxicity test. (A) Morphology of L929 cells in the control and experimental groups on days 3 and 5 (magnification, x100). The cells in control group were incubated with DMEM only, while those in experimental groups were treated with DMEM soaked with the gel for $24 \mathrm{~h}$. (B) Cytotoxicity of chitosan temperature-sensitive gel membrane extract liquid on L929 cells determined by an MTT assay.

Chitosan temperature-sensitive gel prolongs the contact duration of levofloxacin with the eye. To examine the eye retention of chitosan temperature-sensitive gel, fluorescein sodium was added into the levofloxacin drug loading system in groups 1-3. The results indicated that the retention time of

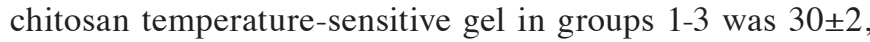
$480 \pm 25$ and $540 \pm 36 \mathrm{~min}$, respectively, which was consistent with the results of the in vitro release assay (data not shown). This result indicated that the chitosan temperature-sensitive gel markedly prolongs the contact duration of levofloxacin with the eye.

Chitosan temperature-sensitive gel is safe and provides good biocompatibility. To evaluate the safety of chitosan temperature-sensitive gel and the biocompatibility it provides, a cell compatibility test, a cytotoxicity test and an ocular skin irritation test were performed. The corneal endothelial cells grown on top of the hydrogel in the control and experimental groups had pebble-like shapes and had formed dense monolayers by day 5 (Fig. 4A). An MTT assay demonstrated that the number of corneal endothelial cells that were treated with DMEM soaked with the hydrogel in the experimental group was insignificantly higher than that in the control group on days 3 and 5 ( $\mathrm{P}>0.05$ ), suggesting that chitosan-based hydrogel membrane had good cytocompatibility (Fig. 4B). Microscopic observation indicated that the growth of L929 cells in the control and experimental groups was not significantly different (Fig. 5A). Consistently with this, the MTT cytotoxicity assay indicated that the 


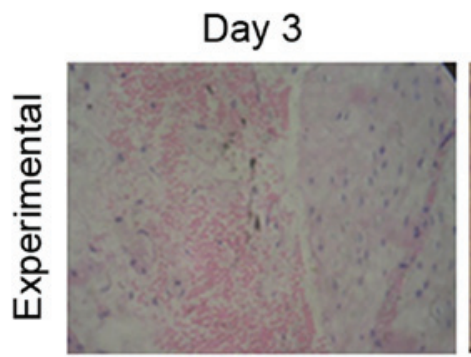

Day 3

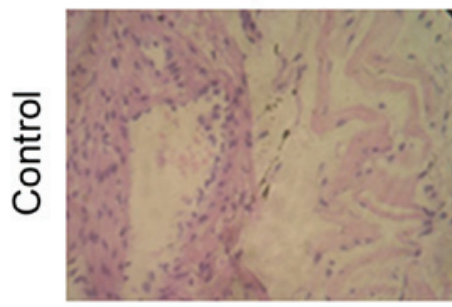

Day 5

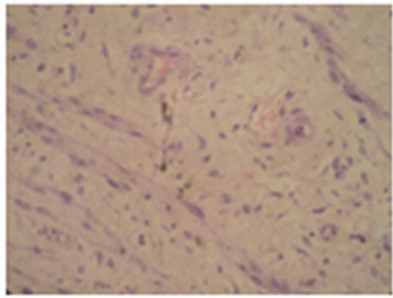

Day 5

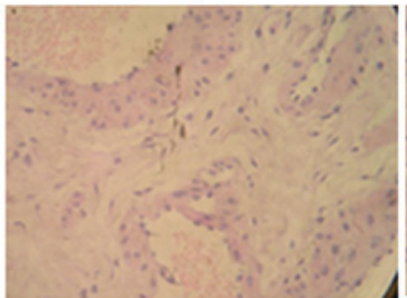

Day 7

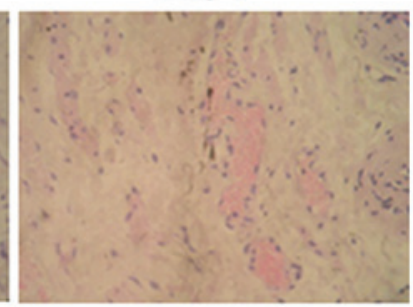

Day 7

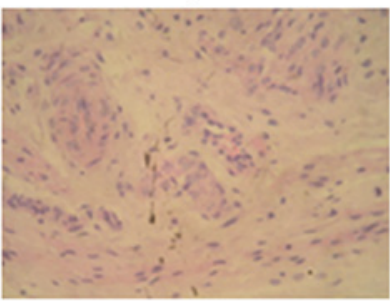

Figure 6. Skin irritation test. A sterile and pyrogen-free chitosan-based temperature-sensitive hydrogel membrane (5 g) was soaked in $5 \mathrm{ml}$ saline for $72 \mathrm{~h}$ Saline $(0.1 \mathrm{ml})$ was injected subcutaneously into six points in the control group, and DMEM soaked in chitosan-based temperature-sensitive hydrogel (0.1 ml) was injected subcutaneously into the six points in experimental groups. At 12, 24 and $48 \mathrm{~h}$ after injection, each injection point of each rabbit was evaluated to determine the artificial vascular stimulation fraction. On days 3, 5 and 7 after surgery, subcutaneous tissues were obtained from all animals and subjected to hematoxylin and eosin staining (magnification, $\mathrm{x} 10$ ).

absorbance in the experimental group was insignificantly higher than that in control group ( $\mathrm{RGH} \geq 100 \%)$, suggesting that the chitosan temperature-sensitive gel had a cytotoxicity of grade 0 (Fig. 5B). The skin irritation test indicated that the control and experimental groups had nearly no congestion phenomenon and the experimental group scored 0. After stimulation for 48, 96 or $144 \mathrm{~h}$, the rabbits in the two groups did not display any behavioral abnormalities. No erythematous response was observed during the entire course of the experiment. H\&E staining of the injection sites on days 3,5 and 7 was not indicative of any inflammatory response (Fig. 6), suggesting that chitosan temperature-sensitive gel caused nearly no skin irritation. These results suggested that chitosan temperature-sensitive gel is safe and provides good bioavailability.

\section{Discussion}

Chitosan has been widely assessed for use in drug delivery systems due to its biocompatibility, safety and other unique properties. In addition, it is included in the usage standards for food additives as a thickening or coating agent (16). At present, research on chitosan and its derivatives in ophthalmic drug delivery systems still mainly focus on improving the solubility of chitosan, increasing the drug load and enhancing the stability of preparations (17). However, only few studies have focused on the absorption, transportation, distribution and metabolization of drugs after administration (13). In the present study, the optimal proportions of the major components and manufacturing process of chitosan temperature-sensitive gel were determined, and the physical and chemical properties, the slow-release effect and the safety of chitosan temperature-sensitive gel loaded with drug-containing nanoparticles were investigated.

The $\mathrm{pH}$ value of artificial tears is $\sim 8$ and their addition is equivalent to that of crosslinking agent. As a result, the temperature-sensitive system easily solidifies into a gel $(17,18)$. In the present study, a temperature-sensitive in situ gel loaded with levofloxacin-containing microspheres was prepared. Chitosan microspheres easily attach to cornea and have a slow-release effect. Therefore, the retention time of drugs on the cornea is prolonged, the local bioavailability of drugs on the eye surface is enhanced and adverse effects of the drugs are reduced $(19,20)$.

During the preparation of levofloxacin chitosan microspheres via the ionic gelation method in the present study, levofloxacin molecules become surrounded by a network structure formed by chitosan and sodium tripolyphosphate $(6,21,22)$. The particle size of the microspheres is in a range that meets the requirements of an ophthalmic particle dispersion system. The measured particle size determined by a particle sizing meter in the present study is the size of hydrated particles, while that determined by scanning electron microscopy is the size of dried particles. As a result, the particle size determined by scanning electron microscopy was smaller. In addition, the zeta potential suggests that the system is stable, and guaranteed the quality of the prepared temperature-sensitive in situ gel.

Chitosan nanoparticles have biological adhesion properties and may attach to conjunctival cells $(9,23,24)$. As a result, the tight junction between epithelial cells of the cornea and conjunctiva may be transiently opened to increase the permeability of the drug in the cornea and conjunctiva. The in vitro slow release and ocular retention experiments demonstrated that chitosan temperature sensitive gel loaded with levofloxacin microspheres prolonged the contact duration between the microspheres and eyes, and ensured the steady and sustained release of the drug. Chitosan temperature-sensitive hydrogel must not have any adverse effects on the organizational structure of organisms when being in contact with tissues in vivo $(25,26)$. The cell compatibility test, the cytotoxicity test 
and the skin irritation test in the present study have demonstrated that chitosan-based temperature-sensitive hydrogel has good biocompatibility and safety. No adverse effects are observed. However, a limitation of the present study was that only an extract of the gel was applied to the cells. To conclude, the present study provided a basis for the future development of the use of chitosan-based temperature-sensitive hydrogel in ophthalmic drug delivery.

\section{Acknowledgements}

This study was supported by the Shandong Technology Development Project (grant no. 2014GHY115025), the Qingdao Science and Technology Bureau (grant no. 103315-NSH) and the National Natural Science Foundation of China (grant no. 31071014).

\section{References}

1. Barros SC, Silva AA, Costa DB, Costa CM, Lanceros-Méndez S, Maciavello MNT, Ribelles JLG, Sentanin F, Pawlicka A and Silva MM: Thermal-mechanical behaviour of chitosan-cellulose derivative thermoreversible hydrogel films. Cellulose 22 1911-1929, 2015.

2. Sandra CB, Ana AS, Diana BC, Ivana C, Carlos MC, Senentxu LM, Agnieszka P and Maria MS: Thermo-sensitive chitosan-cellulose derivative hydrogels: Swelling behaviour and morphologic studies. Cellulose 21: 4531-4544, 2014.

3. Bashari A, Hemmatinejad N and Pourjavadi A: Hydrophobic nanocarriers embedded in a novel dual-responsive poly $(\mathrm{N}$-isopropylacrylamide $) /$ chitosan/( $\beta$-cyclodextrin) nanohydrogel. J polym Res 20: 256, 2013.

4. Luo Q, Gao H, Peng L, Liu G and Zhang Z: Synthesis of PEGylated chitosan copolymers as efficiently antimicrobial coatings for leather. J Appl Polym Sci 133: 2016. doi: 10.1002/app.43465.

5. Ji QX, Zhao QS, Deng J and Lü R: A novel injectable chlorhexidine thermosensitive hydro gel for per iodontal application: Preparation, antibacterial activity and toxicity evaluation. J Mater Sci Mater Med 21: 2435-2442, 2010.

6. Jiang Y, Meng X, Wu Z and Qi X: Modified chitosan thermosensitive hydrogel enables sustained and efficient anti-tumor therapy via intratumoral injection. Carbohydr Polym 144: 245-253, 2016

7. Gratieri T, Gelfuso GM, Rocha EM, Sarmento VH, de Freitas O and Lopez RF: A poloxamer/chitosan in situ forming gel with prolonged retention time for ocular delivery. Eur J Pharm Biopharm 75: 186-193, 2010.

8. de la Fuente M, Raviña M, Paolicelli P, Sanchez A, Seijo B and Alonso MJ: Chitosan-based nanostructures: A delivery platform for ocular therapeutics. Adv Drug Deliver Rev 62: 100-117, 2010.

9. Xu FZ, Zhao TQ, Yang T, Dong LL, Guan XY and Cui XJ: Fabrication of folic acid functionalized $\mathrm{pH}$-responsive and thermosensitive magnetic chitosan microcapsules via a simple sonochemical method. Colloids Surf A Physicochem Eng Asp 490: 22-29, 2016.

10. Abdelbary G: Ocular ciprofloxacin hydrochloride mucoadhesive chitosan-coated liposomes. Pharm Dev Technol 16: 44-56, 2011.

11. Dudhani AR and Kosaraju SL: Bioadhesive chitosan nanoparticles: Preparation and characterization. Carbohydr Polym 81 243-251, 2010.
12. Cao Y, Zhang C, Shen W, Cheng Z, Yu LL and Ping Q: Poly (N-isopropylacrylamide)-chitosan as thermosensitive in situ gel-forming system for ocular drug delivery. J Control Release 120: 186-194, 2007.

13. Madhugiri PV, Purohit KL, Teggin MPK and Hosakote GS: In situ gels based drug delivery systems. Curr Drug Ther 6: 213-222, 2011.

14. Niranjan R, Koushik C, Saravanan S, oorthi A Vairamani M and Selvamurugan N: A novel injectable temperature-sensitive zinc doped chitosan $/ \beta$-glycerophosphate hydrogel for bone tissue engineering. Int J Biol Macromol 54: 24-29, 2013.

15. Yang Y, Liu Q, Guo Y, Wang C, Fu T, et al: The viability and property of rabbit corneal endothelial cells by spheroid culture. Chin J Exp Ophthalmol 32: 786-790, 2014.

16. Chen SL, Liu MZ, Jin SP and Chen Y: pH-/temperaturesensitive carboxymethyl chitosan/poly(N-isopropylacrylamide-co-methacrylic acid) IPN: Preparation, characterization and sustained release of riboflavin. Polym Bull 71: 719-734, 2014.

17. Zhao SP, Zhou F and Li LY: pH- and temperature-responsive behaviors of hydrogels resulting from the photopolymerization of allylated chitosan and $\mathrm{N}$-isopropylacrylamide and their drug release profiles. J Polym Res 19: 9944, 2012.

18. Jude IN, Luke MG, John K and Clement LH: Development of novel chitosan-poly(N,N-diethylacrylamide) IPN films for potential wound dressing and biomedical applications. J Polym Res 20: 161, 2013.

19. Zhou HY, Jiang LJ, Cao PP, LI JB and Chen XG: Glycerophosphate-based chitosan thermosensitive hydrogels and their biomedical applications. Carbohydr Polym 117: 524-536, 2015.

20. Tsai CY, Wong LC, Yen JC, Tseng PC, Chiou SH, Sung YJ, Liu KT and Cheng YH: Thermosensitive chitosan-based hydrogels for sustained released of ferulic acid on corneal wound healing. Carbohydr Polym 135: 308-315, 2016.

21. Zhou HY, Wang ZY, Duan XY, Jiang LJ, Cao PP, Li JX and Li JB: Design and evaluation of chitosan- $\beta$-cyclodextrin based thermosensitive hydrogel. Biochem Eng J 111: 100-107, 2016.

22. Baei P, Jalili-Firoozinezhad S, Rajabi-Zeleti S, Tafazzoli-Shadpour M, Baharvand $\mathrm{H}$ and Aghdami N: Electrically conductive gold nanoparticle-chitosan thermosensitive hydrogels for cardiac tissue engineering. Mater Sci Eng C Mater Biol Appl 63: 131-141, 2016.

23. Modrzejewska Z, Skwarczyńska A, Douglas TEL, Biniaś D, Maniukiewicz W and Sielski J: Structure of chitosan gels mineralized by sorption. J Mol Struct 1098: 101-109, 2015.

24. Cheng YH, Tsai TH, Jhan YY, Chiu AW, Tsai KL, Chien CS, Chiou SH and Liu CJ: Thermosensitive chitosan-based hydrogel as a topical ocular drug delivery system of latanoprost for glaucoma treatment. Carbohydr Polym 144: 390-399, 2016.

25. Assaad E, Maire M and Lerouge S: Injectable thermosensitive chitosan hydrogels with controlled gelation kenetics and enhanced mechanical resistance. Carbohydr Polym 130: 87-96, 2015.

26. Qi X, Qin X, Yang R, Qin J, Li W, Luan K, Wu Z and Song L: Intra-articular administration of chitosan thermosensitive in situ hydrogels combined with diclofenac sodium-loaded alginate microspheres. J Pharm Sci 105: 122-130, 2016.

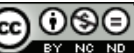

This work is licensed under a Creative Commons Attribution-NonCommercial-NoDerivatives 4.0 International (CC BY-NC-ND 4.0) License. 